\title{
NEUTRON EVAPORATION FROM THE SURFACE OF NEUTRON STARS
}

\author{
K. TENNAKONE \\ Department of Pbysics, Rubuna University College, Matara, Sri Lanka.
}

(Date of receipt : 1 April 1985)

(Date of acceptance: 9 July' 1985)

\begin{abstract}
It is shown that a newly horn neutron star could cool more rapidly by neutron evaporation thin through neutrino emission. The mass loss due to neutron evaporation is estimated.
\end{abstract}

\section{Introduction}

In current supernova scenarios a neutron star is born in a highly excited state, with an energy of excitation exceeding the energy of the final equilibrium state by more than $10^{53}$ ergs, ie. a temperature at birth few times $10^{11}{ }^{0} K^{1,3}$ It is generally believed that this excitation is dissipated by neutrino and antineutrino emission. ${ }^{4,5}$ In this note, it is pointed out that around temperatures of the order $10^{11}{ }^{\circ} \mathrm{K}$ a more efficient mechanism for cooling is evaporation of neutrons. It is shown that a newly born neutron star at a temperature $\sim 10^{11}{ }^{\circ} \mathrm{K}$ will emit a burst of neutrons lasting for about 1 sec and cools' to a temperature $\approx 10^{10}{ }^{\circ} \mathrm{K}$.

\section{The Equations}

We assume that neutrons in the star behave as a noninteracting nearly degenerate fermi gas. Since the neutron star matter is highly conducting, the surface temperature immediately after birth could also be few times $10^{11}{ }^{\circ} \mathrm{K}\left(10^{11}{ }^{\circ} \mathrm{K} \simeq 10 \mathrm{MeV}\right)$. At these temperatures the matter at the surface of the star will consist predominantly of neutrons. Neutrons in the surface region having energies near the fermi level can leave the star if they have sufficient kinetic energy to overcome the potential barrier resulting from nuclear and gravitational forces. Because of the strong interaction; the energy $\Phi_{n}$ necessary to evaporate a neutron from the surface must at most be of the order of binding energy per nucleon of the most stable nucleus $56 \mathrm{Fe} \sim 10 \mathrm{MeV}$. To escape from the gravitational confinement of the star, the evaporated neutrons must do an additional amount of work,

$$
\Phi_{\mathrm{g}}=\mathrm{GM} / \mathrm{R}
$$

where $\mathrm{G}=$ gravitational constant, $\mathrm{M}=$ mass; $\mathrm{K}=$ radius of the star. $\mathrm{F}$ or a neutron star of $1 \mathrm{M}$ and radius $10 \mathrm{~km}, \dot{\Phi}_{\mathrm{g}} \simeq 14 \mathrm{MeV}$. 
Thus the total work function is,

$$
\Phi=\Phi_{\mathrm{n}}+\Phi_{\mathrm{g}} \quad 24 \mathrm{MeV} .
$$

The flux of evaporated neutrons leaving the surface can be calculated as follows. The rate at which the neutrons in the momentum range $p+d p$ and $p$ will strike unit area on the surface of the star near any point $\mathbf{P}$ is,

$$
\left(4 \pi^{3} \hbar^{3}\right)^{-1} \mathrm{fv}_{\mathrm{x}} d p
$$

where $\mathrm{f}=$ fermi distribution function, $\mathrm{v}_{\mathrm{x}}=$ the component velocity of a neutron in the radial direction (chosen to be the $\mathrm{x}$ direction) at $\mathrm{P}$. Neutrons with normal component of momentum,

$$
\mathrm{P}_{\mathrm{x}}>\mathrm{P}_{\mathrm{O}}=2 \mathrm{~m}(\Phi+\mu)^{1 / 2},
$$

will escape from the star. Hence the radial current density leaving the surface of the star is,

$J=\left(4 \pi^{3} n^{3} m\right)^{-1} \int_{-\infty}^{\infty} \int_{-\infty}^{\infty} \int_{p_{0}}^{\infty}[\operatorname{Exp}(\mu-1) / k T+1]^{-1} p_{x} d p_{x} d p_{y} d p_{z}$

where $E=1 / 2 m\left(p_{x}^{2}+p_{y}^{2}+p_{z}^{2}\right), \quad \mu=$ chemical potential characterizing the distribution $f$. Performing the first integration in (2) we get,

$J=\left(4 \pi^{3} n^{3}\right)^{-1} k T \int_{-\infty}^{\infty} \int_{-\infty}^{\infty} \ln \left(1+e^{-s}\right) d p_{y} d p_{z}$

where

$$
\mathrm{s}=1 / \mathrm{kT} \quad\left[\Phi+\left(\mathrm{p}_{\mathrm{y}}^{2}+\mathrm{p}_{\mathrm{z}}^{2}\right) / 2 \mathrm{~m}\right]
$$

Evaluating the resulting double integral in (3) approximately, we obtain,

$$
\mathrm{J}=\mathrm{AT}^{2} \mathrm{e}^{-} \Phi / \mathrm{kT}
$$

where

$$
\mathrm{A}=\left(\mathrm{mk}^{2} / 2 \pi^{2} \hbar^{3}\right)
$$

Emitting neutrons will carry away mass and thermal energy from the star. If $\mathrm{N}=$ number of neutrons in the star at time $\mathrm{t}$ and $\rho=$ density which is assumed to be constant through out the evaporation, then the total flux of neutrons escaping from the star at time $t$ is, 


$$
\begin{aligned}
-\mathrm{dN} / \mathrm{dt} & =4 \pi \cdot \mathrm{AR}^{2} \mathrm{~T}^{2} \mathrm{e}^{-} \Phi / \mathrm{kT} \\
& =4 \pi \mathrm{AT}^{2}(3 \mathrm{mN} / 4 \pi \rho)^{2 / 3} \mathrm{e}^{-\Phi / \mathrm{kT}}
\end{aligned}
$$

For a neutron star of $1 \mathrm{M}_{\mathrm{O}}$ and radius $10 \mathrm{~km}$ at $10^{11}{ }^{\circ} \mathrm{K}$ the expression (5) gives $\mathrm{dN} / \mathrm{dt}=-10^{59} \mathrm{sec}^{-1}$. However, because of cooling and decrease in surface area from loss of mass, the star will not emit neutrons : continously at a rate comparable to the above. It will be shown that a newly born neutron star could emit a flash of neutrons resulting in a significant drop in temperature and loss of mass.

Each emitted neutron will take away from the star an amount of thermal energy equal to its kinetic energy plus the work done against the potential barrier. That is the average energy removed from the star per emitted neutron is $(\langle\mathrm{E}\rangle+\Phi)$. Where $\langle\mathrm{E}\rangle=$ average kinetic energy of the emitted neutrons. The quantity $\langle\mathrm{E}\rangle$ can be calculated by finding the velocity distribution function for the emitted neutrons. To find this velocity distribution function, consider neutrons just inside a small region on the surface of the star. The number of neutrons in this region with the component of velocity normal to the surface (chosen to be the $\mathrm{x}$ component) between $v_{x}+d v_{x}$ and $v_{x}$ is,

$\mathrm{n}\left(\mathrm{v}_{\mathrm{x}}\right)=\left(4 \pi^{3} \hbar^{3}\right)^{-1} \mathrm{mdv} \mathrm{x}_{-\infty} \int_{-\infty}^{\infty} \int_{-\infty}^{\infty} \mathrm{dv}_{\mathrm{y}} \mathrm{d} \mathrm{v}_{\mathrm{z}}[\operatorname{Exp}(\mathrm{E}-\mu) / \mathrm{kT}+1]^{-1}$

If $u_{x}$ is the normal component of the velocity of a neutron after emission,

$$
1 / 2\left(\mathrm{mu}_{\mathbf{x}}^{2}\right)=1 / 2\left(\mathrm{mv}_{\mathbf{x}}^{2}\right)-\mu-\Phi
$$

Subsitituting (7) in (6) and evaluating the integral (6) approximately we get, $\mathrm{n}\left(\mathrm{v}_{\mathrm{x}}\right) \mathrm{dv} \mathrm{v}_{\mathrm{x}} \quad\left(\mathrm{m}^{2} \mathrm{kT}\right)\left(2 \ddot{\pi}^{2} \mathrm{~h}^{3}\right)^{-1} \mathrm{Exp}-\left(\mathrm{mu}_{\mathrm{x}}^{2} \cdot i 2 \mathrm{kT}-\Phi / \mathrm{kT}\right) \mathrm{du}$

and the velocity distribution function for the radial component of the velocities of the emitted neutrons is,

$$
f\left(u_{x}\right) d u_{x}=\left(m u_{x} / k T\right) \operatorname{Exp}-\left(m u_{x}^{2} / 2 k T\right) d u_{x}
$$

and the average kinetic energy of neutrons associated with the radial component of the velocities is,

$$
\begin{aligned}
<\mathrm{mu}_{\mathrm{x}}^{2} / 2 & >=\mathrm{m} / 2 \int_{0}^{\infty} \mathrm{f}\left(\mathrm{u}_{\mathrm{x}}\right) \mathrm{u}_{\mathrm{x}}^{2} \mathrm{du}_{\mathrm{x}}^{2} \\
& =\mathrm{kT}
\end{aligned}
$$


The component velocities parallel to the surface do not change upon emission ie,

$$
\left\langle\mathrm{mu}_{\mathrm{y}}^{2} / 2\right\rangle=\left\langle\mathrm{mu}_{\mathrm{z}}^{2} / 2\right\rangle=\mathrm{kT} / 2
$$

Therefore $\langle\mathrm{E}\rangle=2 \mathrm{kT}$ and the average energy carried away per neutron emitted is $(2 \mathrm{kT}+\Phi)$. If $\mathrm{U}$ is the heat content of the star, the rate of dissipation of thermal energy by neutron emission is,

$$
-\mathrm{W}_{\mathrm{n}}=\mathrm{dU} / \mathrm{dt}=\mathrm{dn} / \mathrm{dt}(2 \mathrm{kT}+\Phi)
$$

Using (5) the above result can also be written as,

$$
\mathrm{W}_{\mathrm{n}}=4 \pi \mathrm{AR}^{2} \mathrm{~T}^{2}(2 \mathrm{kT}+\Phi) \mathrm{e}^{-\Phi / \mathrm{kT}}
$$

Now we compare the cooling due to neutron emission with that due to neutrino emission. The neutrino processes important in the initial cooling of neutron stars is supposed to be the modified URCA and the inverse process ${ }_{4}^{4}$ and the neutrino luminosity from the above processes is estimated to be 4

$$
\mathrm{W}_{v} \quad \alpha \mathrm{T}^{8}
$$

where $\alpha=3 \times 10^{-67} \mathrm{M}\left(\rho_{\mathrm{n}} / \rho\right)^{1 / 3}, \rho_{\mathrm{n}}=$ nuclear matter density. The Table 1 gives $\mathrm{W}_{\mathrm{n}}$ (equation 13) and $\mathrm{W}_{v}$ (equation 14) at different temperatures for a neutron star $1 \mathrm{~m}_{\mathrm{O}}$ and radius $10 \mathrm{~km}$. It is seen at temperatures close to $10^{11}{ }^{\circ} \mathrm{K}$ the energy carried away by neutrons is comparable to the losses due to neutrino and antineutrino emission, (at these temperatures the energy lost. from the surface as thermal radiation is $\left.\sim 10^{41} \quad \operatorname{erg} \sec ^{-1}<<\mathrm{W}_{\mathrm{n}}\right)$. The rate of cooling and mass lost due to neutron emission can also be determined easily. It turns out that at tempera-

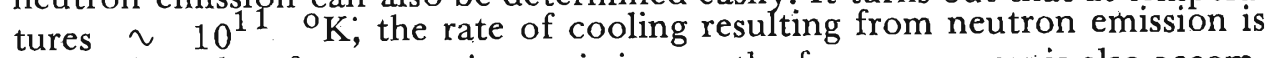
faster than that from neutrino emission, as the former process is also accompanied by a change in volume of the star.

The heat content of the star is the same as that of a degenerate fermi gas ie,

$$
\mathrm{U}=\pi^{2} \mathrm{k}^{2} \mathrm{~T}^{2} \mathrm{~N} / 4 \mu
$$

Thus equation (12) becomes,

$$
\left(\pi^{2} \mathrm{k}^{2} / 4 \mu\right) \mathrm{d} / \mathrm{dN}\left(\mathrm{T}^{2} \mathrm{~N}\right)=2 \mathrm{kT}+\Phi
$$

The solution of equation (16) with the initial condition $N=N_{O}$ when $\mathrm{T}=\mathrm{T}_{\mathrm{O}}\left(\mathrm{T}, \mathrm{T}_{\mathrm{O}}<\mathrm{a}\right)$ is, 


$$
N=N_{0}\left(a-T_{0}\right)(a-T)^{-1}\left(b+T_{0}\right)(b+T)^{-1}
$$

where $\mathrm{a}=8 \mu / \pi^{2} \mathrm{k}, \mathrm{b}=\Phi / 2 \mathrm{k}(\mu \sim 100 \mathrm{MeV})$. Substituting (17) into (14) we get after some approximations,

$$
\mathrm{dT} / \mathrm{dt} \quad-\mathrm{BT}^{2} \mathrm{e}^{-\Phi / \mathrm{kT}}
$$

where $\mathrm{B}=\mathrm{mk}^{2} / \mathrm{n}^{3} \cdot(3 \mathrm{~m} / 4 \pi \rho)^{2 / 3}\left(\mathrm{a}+\mathrm{b}+2 / 3 \mathrm{~T}_{\mathrm{O}}\right) \mathrm{N}_{\mathrm{o}}^{-1 / 2}$

Equation (18) describes the rate of cooling of the star due to neutron emission and solving (18) with the initial condition $t=0, T=T_{o}$ we obtain,

$$
\mathrm{t}=\mathrm{k} / \mathrm{B} \Phi\left(\mathrm{e}^{\Phi / \mathrm{kT}}-\mathrm{e}^{\Phi / k T} \mathrm{o}\right)
$$

The above equation indicates that a neutron star of $1 \mathrm{M}_{\mathrm{O}}$ and radius $10 \mathrm{~km}$ at $10^{11}{ }^{\circ} \mathrm{K}$, cools to $10^{10}{ }^{\circ} \mathrm{K}$ in about $1 \mathrm{sec}$. It takes much longer to cool through the same temperature range via neutrino emission. From (14) and (15) we obtain the equation

$$
\pi^{2} \mathrm{k}^{2} / 4 \mu \mathrm{d} / \mathrm{dt}\left(\mathrm{T}^{2} \mathrm{~N}\right)=-\alpha \mathrm{T}^{8}
$$

that describes cooling of the star due to neutrino emission. The solution of (21) ie,

$$
\mathrm{t}=\left(\pi^{2} \mathrm{k}^{2} \mathrm{~N} / 2 \mu \alpha\right)\left(\mathrm{T}^{-6}-\mathrm{T}_{\mathrm{o}}^{-6}\right)
$$

shows that a neutron star of $1 \mathrm{M}_{\mathrm{O}}$ and radius $10 \mathrm{~km}$ takes $\sim 10^{3} \mathrm{sec}$ to cool through the same temperature range. At lower temperatures $(\mathrm{kT}<<\Phi)$ cooling from neutron emission becomes insignificant because of the damping of the rate of cooling by the exponential factor in (18). The above analysis indicates that in the temperature range $10^{11}-10^{10}{ }^{\circ} \mathrm{K}$, the cooling is predominantly from emission of neutrons. In this temperature range, the rate of neutron evaporation is only slightly affected by neutrino cooling and a significant portion of the mass of the star is lost as evaporating neutrons. 


\begin{tabular}{|l|c|c|}
\hline $\mathbf{T}^{\mathbf{0}} \mathrm{K}$ & $\mathbf{W}_{\mathbf{n}} \operatorname{erg~sec}^{-1}$ & $\mathbf{W}_{v} \operatorname{erg~sec}^{-1}$ \\
\hline $10^{9}$ & $2.8 \times 10^{-36}$ & $5 \times 10^{38}$ \\
$10^{10}$ & $2.8 \times 10^{43}$ & $5 \times 10^{46}$ \\
$10^{11}$ & $5.5 \times 10^{53}$ & $5 \times 10^{54}$ \\
$10^{12}$ & $1.4 \times 10^{56}$ & $5 \times 10^{62}$ \\
\hline
\end{tabular}

\section{Table 1}

\section{Conclusions}

The conclusion we draw from the above discussion is that a neutron star $\sim 1 \mathrm{M}_{\mathrm{O}}$ and radius $\sim 10 \mathrm{~km}$ will emit a flash of neutrons immediately after its birth, if the surface temperature is around $10^{11}{ }^{\circ} \mathrm{K}$. The duration of the flash is about $1 \mathrm{sec}$ and approximately $10 \%$ of the mass of the star gets evaporated. As the neutron flash is emitted just after the birth, it should interact with matter ejected in the supernova explosion (neutron emission will probably continue until the outer shell of ejected matter has moved to a distance $10^{3}-10^{4} \mathrm{~km}$ ). This effect could have important consequences, neutron and proton (protons from decay of emitted neutrons) bombardment of the ejected matter may be efficient in synthesising; heavy elements. ${ }^{6}$ The flux of protons may also be a source of cosmic rays.

It is very important to note that the process depends entirely on evaporation of neutrons from the surface and does not involve mass transport from inside. The star wears away from the surface due to loss of neutrons. 


\section{References}

1. ARNETT, W. D. (1968) Astrophys. J, $153: 341$

2. BROWN, G. E. (1977) Comments on Astropbys., $7: 67$

3. BAHCALL, J. N. \& WOLF, R. A. (1965) Pbys. Rev. 140B : 1445

4. COlgate, S. A. \& WHITE, R. H. (1966) Astropbys. J, $146: 626$

5. SAWYER, R. F. \& SONI, A. (1979) Astrophys. J, $222: 286$

6. TRURN, J. W. (1969) Supernova and their Ramnants ed by BRANCAZIO, P. J. \& CAMERON, A. G. W. (Gordon \& Breach) 